\title{
Accroissement et qualité des tiges de deux jeunes futaies au Témiscouata
}

par

Chhun-Huor Ung ${ }^{1}$ et Jacques Trencia ${ }^{1}$

\section{Résumé}

L'estimation de l'accroissement annuel courant en volume des arbres et l'évaluation de la qualité de leur tige permettent de conclure que les deux jeunes futaies ont une valeur potentielle pour la production du bois de sciage et donc de justifier la rentabilité des coupes d'assainissement qui devront s'y réaliser.

L'accroissement annuel courant en volume s'estime par le sondage à la tarière des arbres dans chacune des placettes-échantillons à nombre d'arbres fixe installées de façon systématique dans les deux massifs. L'évaluation de la qualité des tiges se fait sur les deux premières billes de 2,5 m des arbres sondés.

Mots clés: accroissement annuel courant, taux d'accroissement, qualité des tiges, aménagement intensif.

\begin{abstract}
Current annual increment volume and stem quality evaluation in two high forest stands allowed us to conclude that they have a potential to produce sawlog wood and therefore justify the profitability of the sanitation thinning.

Current annual increment volume was evaluated by extracting increment cores from trees in sample plots set up systematically in the two stands. Stem quality was evaluated on the $2.5 \mathrm{~m}$-logs of the sampled trees.
\end{abstract}

Key words: current annual increment, increment rate, stem quality, intensive management.

\section{Introduction}

Grâce à l'entente avec le Groupement forestier de l'Est du Lac Témiscouata, le Centre de foresterie des Laurentides a acquis depuis 1981 le droit d'effectuer des travaux de recherche pour fins d'aménagement intensif dans deux massifs boisés situés dans le comté de Témiscouata (Québec) à l'extrême nord de la zone des feuillus tolérants.

L'influence humaine exercée sur les forêts du Témiscouata est très importante. Dès le début du $19^{\mathrm{e}}$ siècle, des compagnies d'exploitation forestière détenaient de vastes concessions afin d'alimenter les papeteries. Depuis, les coupes de bois se sont poursuivies à outrance. Dans plusieurs endroits, l'érablière se trouve dégradée avec du bois de faible qualité.

Les objectifs du présent travail consistent à:

- comparer le taux d'accroissement en volume selon le massif, l'essence, la région forestière, selon la classe de cime et selon le site;

- comparer l'accroissement annuel courant en volume à I'hectare selon le massif et la région forestière;

- évaluer la qualité des tiges dont la valeur semble à priori très faible en raison de la dégradation de la forêt.

Les trois aspects du travail ont pour but de vérifier la rentabilité probable des travaux sylvicoles (en particulier les coupes d'assainissement) qui pourront s'y réaliser. Ce travail fait suite à l'analyse de la forme de l'érable à sucre (Acer saccharum Marsh.) et du hêtre à grandes feuilles (Fagus grandifolia Ehrn.) (Ung 1989) dont les résultats ont permis d'établir adéquatement le tarif de cubage des deux essences.

TForêts Canada, Région du Québec, 1055, rue du P.E.P.S., C.P. 3800, Sainte-Foy (Québec) G1V 4C7
Le premier massif appelé Squatec est âgé d'une cinquantaine d'années et a une superficie de 5,9 ha. II est situé à $47^{\circ} 43^{\prime}$ de latitude nord et à $68^{\circ} 34^{\prime}$ de longitude ouest, à $350 \mathrm{~m}$ d'altitude. Le deuxième massif appelé Lejeune est âgé d'une quarantaine d'années et a une superficie de 5,4 ha. II est situé à $47^{\circ} 44^{\prime}$ de latitude nord et à $68^{\circ} 34^{\prime}$ de longitude ouest, à $350 \mathrm{~m}$ d'altitude. Les deux massifs sont situés dans l'extrême est de la zone des feuillus tolérants. La région immédiate des deux peuplements fait partie du domaine climacique de l'érablière à bouleau jaune de Grandtner (1966) et de la région forestière des Grands Lacs et du Saint-Laurent, section L6-Restigouche de Rowe (1972).

L'inventaire intégral des deux massifs a été réalisé en 1982 et 1983. Les placettes-échantillons y ont été installées (48 à Lejeune et 58 à Squatec). C'est dans ces placettes que se sont effectués le sondage à la tarière, l'examen de la qualité des tiges, le relevé de la végétation des strates inférieures et le relevé pédologique.

Le massif Squatec couvre la moitié supérieure d'un versant d'une colline arrondie. Son relief se présente sous forme d'une suite de pentes régulières, courtes et convexes à la manière d'un escalier. La force des pentes varie entre 2 à $10 \%$ avec une exposition vers le sud-ouest. Quant au massif Lejeune, il est situé sur le bas de pente, moyennement longue et régulière, sa force est de $8 \%$ avec l'exposition sud.

\section{Matériel et Méthodes}

\section{Inventaire intégral}

L'inventaire intégral des deux massifs réalisé aux étés 1982 et 1983 a permis d'avoir l'image exacte de leur contenu. Le résultat de cet inventaire servira à l'estimation de l'accroissement annuel courant à l'hectare et à la comparaison du taux d'accroissement selon le site. Le tableau 1 
Tableau 1. Composition des essences dans Lejeune et Squatec: nombre de tiges à l'hectare et volume en $\mathrm{m}^{3} / \mathrm{ha}$ par classes de dhp

\begin{tabular}{|c|c|c|c|c|c|c|c|c|c|c|c|c|}
\hline \multirow[b]{2}{*}{$\begin{array}{l}\text { Classes de } \\
\text { dhp en } \mathrm{cm}\end{array}$} & \multicolumn{6}{|c|}{ Lejeune } & \multicolumn{6}{|c|}{ Squatec } \\
\hline & $\begin{array}{l}\text { Bouleau } \\
\text { jaune }\end{array}$ & $\begin{array}{l}\text { Érable } \\
\text { rouge }\end{array}$ & $\begin{array}{l}\text { Érable } \\
\text { à sucre }\end{array}$ & Hêtre & $\begin{array}{l}\text { Autres } \\
\text { essences* }\end{array}$ & $\begin{array}{l}\text { Toutes les } \\
\text { essences }\end{array}$ & $\begin{array}{l}\text { Bouleau } \\
\text { jaune }\end{array}$ & $\begin{array}{l}\text { Érable } \\
\text { rouge }\end{array}$ & $\begin{array}{l}\text { Érable } \\
\text { à sucre }\end{array}$ & Hêtre & $\begin{array}{l}\text { Autres } \\
\text { essences* }\end{array}$ & $\begin{array}{l}\text { Toutes les } \\
\text { essences }\end{array}$ \\
\hline \multicolumn{13}{|c|}{ Nombre de tiges à l'hectare } \\
\hline jusqu'à 4,9 & 10 & 13 & 273 & 77 & 7 & 380 & 1 & 1 & 738 & 69 & 32 & 841 \\
\hline de 5 à 8,9 & 12 & 14 & 17 & 22 & 3 & 68 & 1 & 2 & 18 & 7 & 3 & 31 \\
\hline de 9 à 19,9 & 144 & 121 & 228 & 170 & 9 & 672 & 7 & 27 & 720 & 50 & 14 & 818 \\
\hline de 20 à 29,9 & 19 & 5 & 14 & 17 & 4 & 59 & 5 & 4 & 147 & 5 & 9 & 170 \\
\hline $\begin{array}{l}\text { de } 30 \text { et plus } \\
\text { toutes les }\end{array}$ & 1 & 0 & 1 & 3 & 1 & 6 & 1 & 0 & 10 & 5 & 13 & 29 \\
\hline classes & 186 & 153 & 533 & 289 & 24 & 1185 & 15 & 34 & 1633 & 136 & 71 & 1889 \\
\hline \multicolumn{13}{|l|}{ Volume en $\mathrm{m}^{3} / \mathrm{ha}$} \\
\hline $\begin{array}{l}\text { Jusqu' a } 4,9 \\
\text { de } 5 \text { à } 8,9\end{array}$ & $\begin{array}{l}0,03 \\
0,25\end{array}$ & $\begin{array}{l}0,03 \\
0,28\end{array}$ & $\begin{array}{l}0,16 \\
0,38\end{array}$ & $\begin{array}{l}0,11 \\
0,46\end{array}$ & $\begin{array}{l}0,01 \\
0,05\end{array}$ & $\begin{array}{l}0,34 \\
1,42\end{array}$ & 0,02 & 0,05 & 0,15 & $\begin{array}{l}0,15 \\
0,15\end{array}$ & 0,06 & $\begin{array}{l}0,24 \\
0,85\end{array}$ \\
\hline de 9 à 19,9 & 18,35 & 13,22 & 23,13 & 17,41 & 0,94 & 73,06 & 0,93 & 3,77 & 87,32 & 4,88 & 1,10 & 98,00 \\
\hline de 20 à 29,9 & 7.01 & 1,70 & 5,05 & 7,19 & 1,58 & 22,54 & 2,09 & 1,66 & 56,48 & 2,31 & 4,04 & 66,58 \\
\hline $\begin{array}{l}\text { de } 30 \text { et plus } \\
\text { toutes les }\end{array}$ & 0,96 & 0,19 & 0,82 & 2,53 & 0,54 & 5,03 & 0,71 & 0,00 & 15,32 & 7.72 & 14,79 & 38,54 \\
\hline classes & 26,60 & 15,42 & 29,54 & 27,70 & 3,12 & 102,39 & 3,75 & 5,48 & 159,84 & 15,14 & 20,00 & 204,21 \\
\hline
\end{tabular}

*Autres essences: bouleau à papier, érable à épis, érable de Pennsylvanie, cerisier de Pennsylvanie, frêne noir, peuplier faux-tremble, épinette blanche, sapin baumier.

montre que Lejeune est constitué essentiellement du mélange de quatre essences: érable à sucre, hêtre, bouleau jaune (Betula alleghaniensis Britton) et érable rouge (Acer rubrum L.), tandis que Squatec est une érablière à sucre presque pure.

Respectivement dans Lejeune et Squatec, 32,1 et $44,5 \%$ des tiges ont des diamètres à hauteur de poitrine (dhp) inférieurs à $5 \mathrm{~cm}$. Pour les deux massifs, la classe 9 à $19,9 \mathrm{~cm}$ est la plus importante; elle représente $71,4 \%$ du volume total à Lejeune avec 672 tiges à l'hectare et $48 \%$ du volume total à Squatec avec 818 tiges à I'hectare.

Les figures 1 et 2 présentent les images de la composition des essences au niveau de chacune des placettes de 10 $\times 10 \mathrm{~m}$ respectivement pour Lejeune et Squatec. II est clair que Lejeune est une mosaïque composée d'érable à sucre, de bouleau jaune et de hêtre tandis que Squatec est une érablière à sucre. Les diverses compositions obtenues sont:

Bétulaie jaune: le bouleau jaune occupe plus de $50 \%$ de la surface terrière totale;

Érablière à sucre: l'érable à sucre occupe plus de $50 \%$ de la surface terrière totale;

Érablière à sucre avec érable rouge: l'érable à sucre et l'érable rouge occupent $50 \%$ de la surface terrière totale; Hêtraie: le hêtre à grandes feuilles occupe plus de $50 \%$ de la surface terrière totale;

Mélangé: aucune essence n'occupe plus de $50 \%$ de la surface terrière totale;

Peupleraie: le peuplier faux-tremble (Populus tremuloides Michx.) occupe plus de $50 \%$ de la surface terrière totale. À noter que lorsqu'une seule tige représente $50 \%$ et plus de la surface terrière totale, c'est l'essence ayant plus de $50 \%$ du nombre total de tiges qui donne le nom à la placette.

\section{Accroissement}

\section{Méthode d'estimation}

Étant donné que le volume est le paramètre dendrométrique le plus complet, nous allons analyser l'accroissement en volume de l'arbre. Les deux types d'accroissement qui seront utilisés sont l'accroissement annuel courant dV/dt et le taux d'accroissement (ou le rapport de l'accroissementannuel courant sur le volume) dV/Vdt.

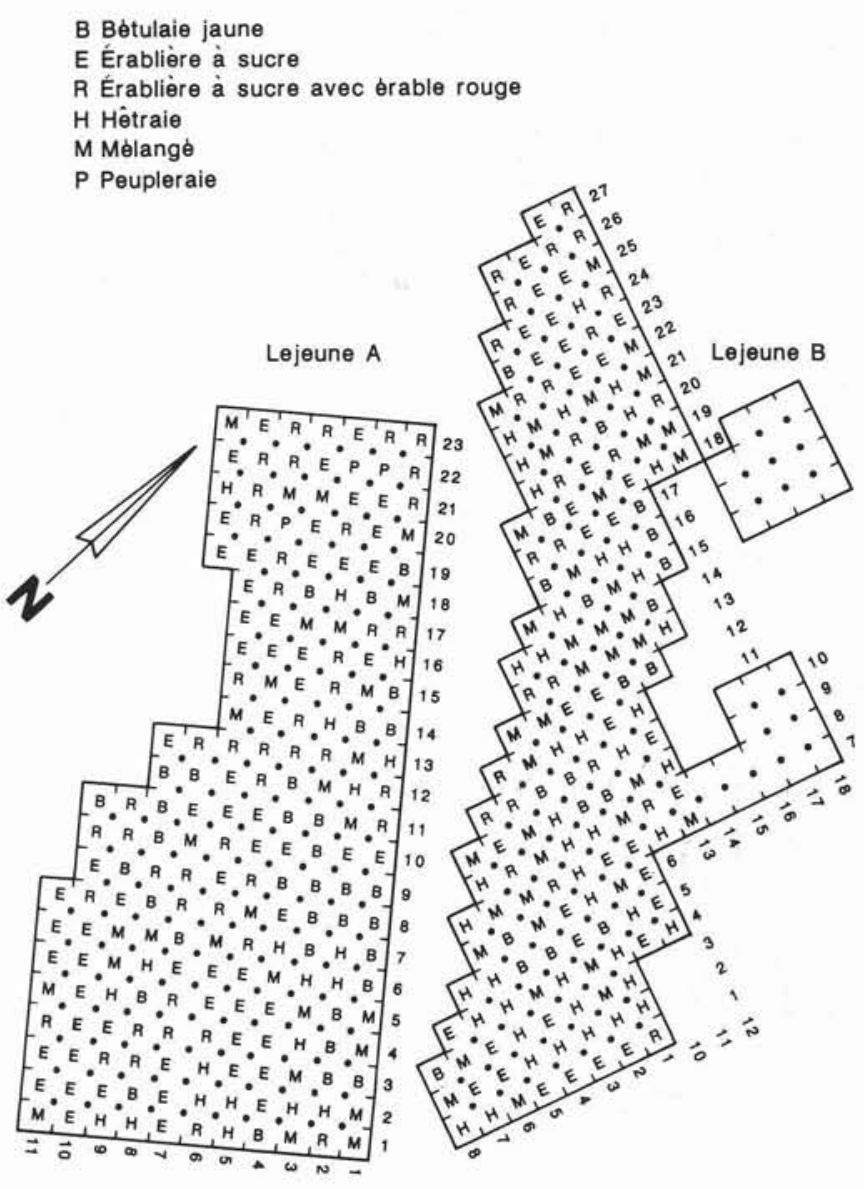

Figure 1. Lejeune: composition des essences au niveau des placettes de $10 \times 10 \mathrm{~m}$. 


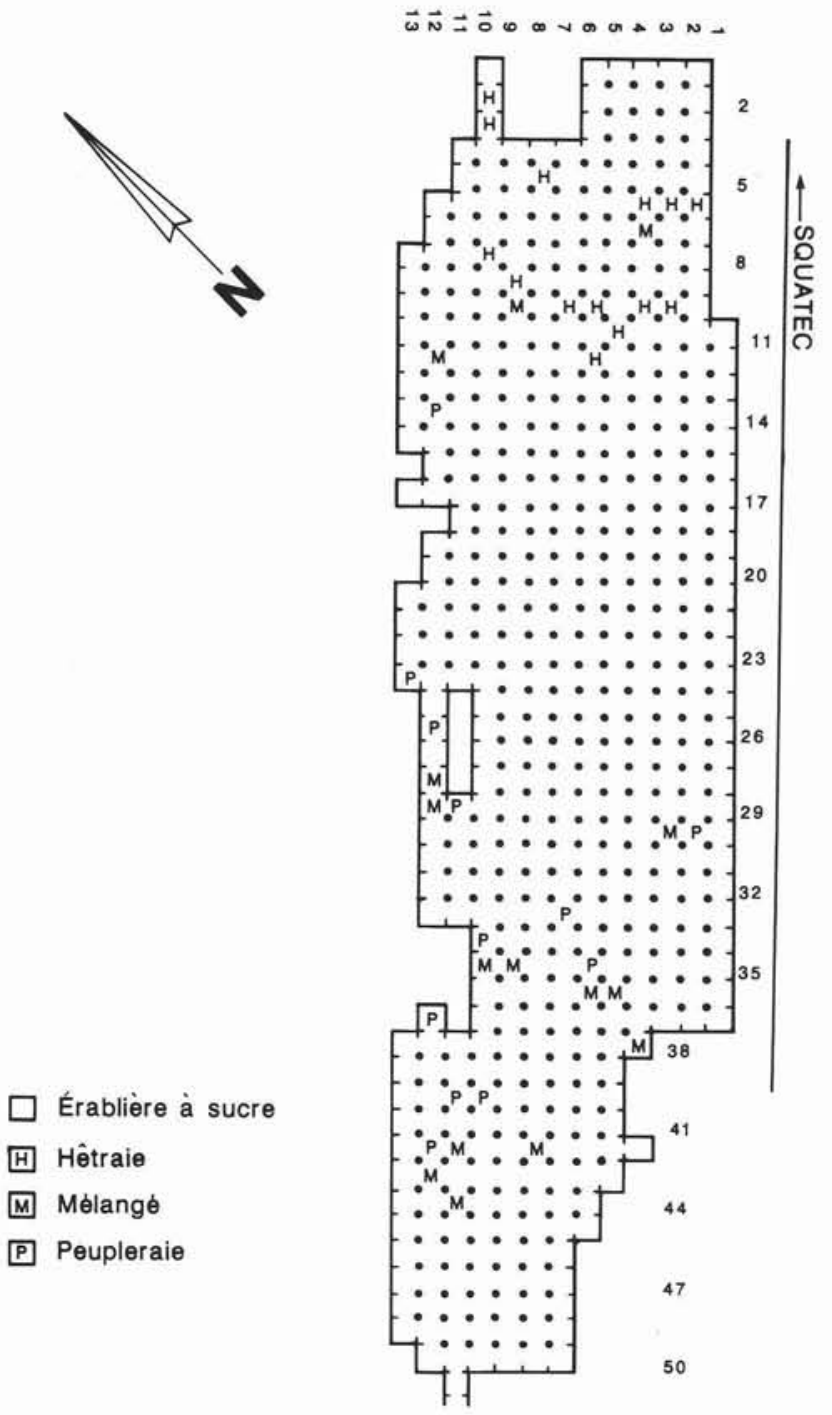

Figure 2. Squatec: composition des essences au niveau des placettes de $10 \times 10 \mathrm{~m}$.

Les estimations de l'accroissement utilisent les résultats des sondages à la tarière et sont fondées sur deux hypothèses (Duplat et Perrotte 1981).

Hypothèse 1. Au moment de la mesure, la loi de la variation du volume d'un arbre en fonction de son diamètre à 1,30 m est exprimée par le tarif de cubage à une entrée applicable à l'essence à laquelle il appartient: $V=f(D)$.

Hypothèse 2. En forêt, les choses évoluent assez lentement pour qu'on puisse considérer que l'accroissement courant en diamètre d'un arbre est bien estimé par la moyenne des accroissements annuels observés sur une courte période passée, cinq ans dans notre cas.

Le principe de la méthode est le suivant. Considérons un arbre dont le diamètre $D$ à $1,30 \mathrm{~m}$ et le volume $\mathrm{V}$ varient en fonction du temps t. À un instant donné, son accroissement courant en volume $\mathrm{dV} / \mathrm{dt}$ est le produit de deux termes. Le premier terme est donné, d'après l'hypothèse 1, par la dérivée du tarif de cubage $V^{\prime}$ (D). Le deuxième terme du produit est l'accroissement courant en diamètre sur écorce de l'arbre à l'instant $t$. Si nous admettons l'hypothèse 2, il peut être estimé par sondage à la tarière. Celui-ci, effectué à 1,30 m de hauteur, est pris perpendiculairement à la section de l'arbre. Le développement mathématique des deux termes permet d'obtenir l'accroissement annuel courant en volume sous la forme:

$\frac{\mathrm{dV}}{\mathrm{dt}}=\frac{2 \mathrm{k}}{\mathrm{W}} \quad \mathrm{LV}^{\prime}(\mathrm{D})$

où

$V^{\prime}(D)$ : dérivée du tarif de cubage pour $D$

$\mathrm{L}$ : longueur occupée par les $\mathrm{W}=5$ derniers cernes

$k=R /(R-e)$ : facteur d'écorce avec $R$ et e représentant respectivement le rayon de l'arbre et son épaisseur d'écorce au moment de la mesure.

\section{Prise des données}

Le sondage à la tarière a été réalisé en août 1986. Une carotte a été prélevée de chacun des arbres situés dans les placettes-échantillons. Le prélèvement des carottes devient impossible pour certains arbres de faibles diamètres.

D'après la répartition des arbres sondés (tableau 2), les essences qui seront étudiées sont: le bouleau jaune, l'érable rouge, l'érable à sucre et le hêtre à grandes feuilles pour Lejeune et seulement l'érable à sucre pour Squatec.

Tarifs de cubage

Grâce aux données d'analyse des tiges abattues à Lejeune, nous avons pu étudier la forme des tiges de l'érable à sucre et du hêtre à grandes feuilles. L'étude a montré que les deux essences réunies permettent d'établir le tarif de cubage suivant:

$V=0,084364(D+1)^{2,65740}$ avec $\mathrm{D}$ sur écorce en $\mathrm{cm}$ et $\mathrm{V}$ en $\mathrm{dm}^{3}$. Ce tarif sera appliqué à l'érable à sucre, au hêtre, mais aussi au bouleau jaune et à l'érable rouge.

Pour le peuplier faux-tremble, nous utilisons le tarif établi par Zarnovican et Ouellet (1987):

$V=0,070058(D+1)^{2,67747}$

avec D sur écorce en $\mathrm{cm}$ et $\mathrm{V}$ en $\mathrm{dm}^{3}$.

Les dérivées de (13) et (14) sont respectivement:

$V^{\prime}=0,224189(D+1)^{1,65740}$
$V^{\prime}=0,187578(D+1)^{1,67747}$

Qualité des tiges

L'accroissement à lui seul ne suffit pas pour juger complètement de la valeur potentielle du bois sur pied. Encore faut-il le compléter par l'examen de sa qualité.

D'après Laszlo (1971), la méthode complète pour évaluer la qualité de l'arbre devrait répondre aux exigences suivantes:

- Être capable de fournir la qualité des arbres sur pied et celle du peuplement;

- Pouvoir être utilisée pour toutes les essences avec de larges fourchettes d'âges et de dimensions et avec n'importe quel type d'aménagement forestier;

- Se baser sur les signes externes de l'arbre; être simple, claire et facile à maîtriser;

- L'examen des signes externes doit se faire sur une plus grande partie possible de la tige;

- Les classes de qualité doivent être de nature à pouvoir 
Tableau 2. Répartition des arbres sondés

\begin{tabular}{lllllll}
\hline & $\begin{array}{l}\text { Bouleau } \\
\text { jaune }\end{array}$ & $\begin{array}{l}\text { Érable } \\
\text { rouge }\end{array}$ & $\begin{array}{l}\text { Érable } \\
\text { à sucre }\end{array}$ & Hêtre & $\begin{array}{l}\text { Peuplier } \\
\text { faux-tremble }\end{array}$ & $\begin{array}{l}\text { Les cinq } \\
\text { essences }\end{array}$ \\
\hline
\end{tabular}

Lejeune

Nombre d'arbres

sondés

Pourcentage

38

13,7
45

16,2
120

43,3
73

26,4
1

0,4
277

100

Squatec

Nombre d'arbres

sondés

Pourcentage

$\begin{array}{cc}3 & 7 \\ 1,1 & 2,7\end{array}$

229

87,4

16

6,1

7

2,7

262

100

se combiner facilement aux possibilités d'évaluation du prix du bois.

Les peuplements étudiés au Témiscouata se composent en majorité d'arbres n'ayant pas encore de valeur marchande. De ce fait, l'exigence relative à l'évaluation du prix du bois n'est pas appropriée pour notre besoin immédiat.

La méthode de Sonderman (1979) est destinée aux jeunes arbres sur pied qui sont justement l'objet de notre étude. L'examen de la qualité se base sur:

- la couronne de l'arbre;

- la courbure du tronc et coude, la fourche, la fente et la blessure;

- le nombre de branches mortes et vivantes.

L'examen de ces caractères se fait sur une longueur de $5 \mathrm{~m}$ divisée en deux billes de $2,5 \mathrm{~m}$. Les valeurs numériques attribuées aux caractères externes sont présentées dans le tableau 3. Lorsque l'arbre est examiné, il peut être classifié dans une des trois qualités suivantes:

\begin{tabular}{lc}
\hline \multicolumn{1}{c}{ Qualité } & Total des valeurs \\
\hline Bonne & 10 à 12 \\
Moyenne & 8 à 9 \\
Médiocre & 1 à 7 \\
\hline
\end{tabular}

En somme, les trois classes de qualité permettent d'identifier, d'une part, les arbres qui n'ont pas de potentiel (qualité médiocre) et d'autre part, ceux qui ont du potentiel pour rentabiliser les traitements sylvicoles (qualités bonne et moyenne).

\section{Résultats et discussion}

\section{Taux d'accroissement}

Comparaison selon le massif, l'essence et la région forestière.

Le tableau 4 présente la moyenne du taux d'accroissement des arbres sondés répartis en cinq classes de dhp.

L'examen des taux d'accroissement révèle certaines différences intéressantes entre peuplements et entre espèces:

$1^{\circ}$ la densité de Lejeune est plus faible que celle de Squatec; ceci se traduit par un accroissement individuel de l'érable à sucre supérieur dans le peuplement de Lejeune;

$2^{\circ}$ les taux de croissance diffèrent selon l'espèce: dans la plus grosse classe diamétrale commune à toutes les essences (20-29, $9 \mathrm{~cm}$ ) le bouleau jaune s'accroît plus rapidement que les autres essences;

$3^{\circ}$ toutes les espèces ne sont pas également nanties pour compétitionner pour la lumière. Trois patrons distincts de variation du taux de croissance selon la grosseur discriminent
Tableau 3. Valeurs numériques attribuées aux divers caractères externes de l'arbre.

\begin{tabular}{llc}
\hline \multirow{2}{*}{ Classe sociale } & Caractères & Valeur \\
\cline { 2 - 3 } & $\begin{array}{l}\text { dominant } \\
\text { codominant } \\
\text { intermédiaire } \\
\text { opprimé }\end{array}$ & 4 \\
& Déviation en cm & 2 \\
\cline { 2 - 3 } Courbure du tronc & 3 & 1 \\
et du coude & 4 à 10 & Valeur \\
& 11 à 15 & 4 \\
& 16 à 20 & 3 \\
& 21 et plus & 2 \\
& & 1 \\
& *Si & $0^{*}$
\end{tabular}

*Si l'arbre a une fourche, une carie ou une fente, la valeur est zéro.

Nombre de branches vivantes et mortes de diamètre supérieur ou égal à $1 \mathrm{~cm}$

\begin{tabular}{lc} 
supérieur ou égal à $1 \mathrm{~cm}$ & Valeur \\
\hline 1 à 2 & 4 \\
3 à 4 & 3 \\
5 à 8 & 2 \\
9 à 16 & 1 \\
17 et plus & 0 \\
\hline
\end{tabular}

Branches vivantes et mortes

le hêtre des érables et du bouleau jaune;

$4^{\circ}$ le patron le plus fréquemment observé est celui du hêtre (Macjen 1979; Fortin 1983): le taux de croissance en volume diminue exponentiellement avec la grosseur;

$5^{\circ}$ les taux d'accroissement des érables augmentent avec la grosseur jusqu'à la classe des $9-19,9 \mathrm{~cm}$. Les taux des individus de la classe supérieure $(20-29,9 \mathrm{~cm})$ sont inférieurs à ceux de la classe précédente même si l'accroissement courant par individu est trois fois plus grand pour les plus gros arbres que pour ceux de 9-19, $9 \mathrm{~cm}$;

$6^{\circ}$ les taux d'accroissement du bouleau jaune augmentent avec la grosseur, de plus les taux des classes de 5 à $20 \mathrm{~cm}$ sont inférieurs à ceux observés pour les autres espèces. Ces deux observations traduisent une perte de vigueur plus forte des codominants et des dominés dans le cas du bouleau jaune que dans celui des autres espèces: la distinction sociale se ferait donc plus rapidement chez le bouleau jaune que chez les érables et d'autre part, plus rapidement chez les érables que chez le hêtre. Une étude précédente (Zarnovican et Trencia 1987) a démontré que les différences d'âges entre individus de ce peuplement étaient très faibles mais que des différences très importantes existaient dans l'accroissement.

À l'intérieur de Lejeune, les quatre essences ont des taux d'accroissement plus ou moins similaires. Pour le bouleau jaune, il convient de signaler son très bon taux d'accroissement dans la classe 20 à $29,9 \mathrm{~cm}$. Mais ceci masque un fait important: le dépérissement des individus de cette espèce sans égard à leur classe sociale. 


\begin{tabular}{|c|c|c|c|c|c|c|}
\hline & \multirow[b]{2}{*}{$\begin{array}{l}\text { Classes de } \\
\text { dhp en cm }\end{array}$} & \multicolumn{4}{|c|}{ Lejeune } & \multirow{2}{*}{$\begin{array}{l}\text { Squatec } \\
\text { Érable } \\
\text { à sucre }\end{array}$} \\
\hline & & $\begin{array}{l}\text { Bouleau } \\
\text { jaune }\end{array}$ & $\begin{array}{l}\text { Érable } \\
\text { rouge }\end{array}$ & $\begin{array}{l}\text { Érable } \\
\text { à sucre }\end{array}$ & Hêtre & \\
\hline $\begin{array}{l}\text { Nombre } \\
\text { d'arbres } \\
\text { sondés }\end{array}$ & $\begin{array}{l}\text { jusqu'à } 4,9 \\
\text { de } 5 \text { à } 8,9 \\
\text { de } 9 \text { à } 19,9 \\
\text { de } 20 \text { à } 29,9 \\
\text { de } 30 \text { et plus }\end{array}$ & $\begin{array}{r}1 \\
1 \\
29 \\
7 \\
0\end{array}$ & $\begin{array}{r}0 \\
4 \\
37 \\
4 \\
0\end{array}$ & $\begin{array}{r}1 \\
29 \\
81 \\
9 \\
0\end{array}$ & $\begin{array}{r}1 \\
12 \\
51 \\
9 \\
0\end{array}$ & $\begin{array}{r}0 \\
19 \\
153 \\
53 \\
1\end{array}$ \\
\hline $\begin{array}{l}\text { Moyenne } \\
\text { du taux } \\
\text { d'accroissement } \\
\text { 100/an }\end{array}$ & $\begin{array}{l}\text { jusqu'à } 4,9 \\
\text { de } 5 \text { à } 8,9 \\
\text { de } 9 \text { à } 19,9 \\
\text { de } 20 \text { à } 29,9 \\
\text { de } 30 \text { et plus }\end{array}$ & $\begin{array}{r}2,73 \\
3,07 \\
4,54 \\
5,56 \\
-\end{array}$ & $\begin{array}{r}-\overline{14} \\
4,76 \\
4,52 \\
-\end{array}$ & $\begin{array}{r}2,43 \\
3,28 \\
4,78 \\
4,68 \\
-\end{array}$ & $\begin{array}{r}10,97 \\
4,44 \\
5,27 \\
3,80 \\
\end{array}$ & $\begin{array}{l}-\overline{2,54} \\
2,80 \\
3,02 \\
3,61\end{array}$ \\
\hline $\begin{array}{l}\text { Écart-type } \\
\text { du taux } \\
\text { d'accroissement } \\
\text { 100/an }\end{array}$ & $\begin{array}{l}\text { jusqu'à } 4,9 \\
\text { de } 5 \text { à } 8,9 \\
\text { de } 9 \text { à } 19,9 \\
\text { de } 20 \text { à } 29,9 \\
\text { de } 30 \text { et plus }\end{array}$ & $\begin{array}{r}- \\
1, \overline{82} \\
0,94 \\
-\end{array}$ & $\begin{array}{r}1, \overline{11} \\
1,77 \\
1,90 \\
-\end{array}$ & $\begin{array}{r}1, \overline{9} \\
2,66 \\
1,54 \\
-\end{array}$ & $\begin{array}{l}-\overline{2,83} \\
1,91 \\
1,90 \\
-\end{array}$ & $\begin{array}{l}-\overline{0,88} \\
1,42 \\
1,53 \\
-\end{array}$ \\
\hline
\end{tabular}

Tableau 5. Comparaison des taux d'accroissement de l'érable à sucre

\begin{tabular}{|c|c|c|c|c|c|c|}
\hline \multirow[b]{2}{*}{$\begin{array}{l}\text { Classes de } \\
\text { dhp en cm }\end{array}$} & \multicolumn{2}{|c|}{ Lejeune } & \multicolumn{2}{|c|}{ Squatec } & \multirow{2}{*}{\begin{tabular}{l}
\multicolumn{1}{c}{ Majcen ${ }^{\star}$} \\
Presler \\
Taux \\
d'accroissement
\end{tabular}} & \multirow{2}{*}{$\begin{array}{l}\text { Fortin** } \\
\text { Taux } \\
\text { d'accroissement }\end{array}$} \\
\hline & $\begin{array}{l}\text { Nombre } \\
\text { d'arbres } \\
\text { sondés }\end{array}$ & $\begin{array}{l}\text { Taux } \\
\text { d'accroissement }\end{array}$ & $\begin{array}{l}\text { Nombre } \\
\text { d'arbres } \\
\text { sondés }\end{array}$ & $\begin{array}{l}\text { Taux } \\
\text { d'accroissement }\end{array}$ & & \\
\hline jusqu'à 4,9 & 1 & 2,43 & 0 & - & & \\
\hline de 5 à 6,9 & 11 & 4,40 & 1 & 2,43 & & \\
\hline de 7 à 8,9 & 18 & 2,59 & 18 & 2,55 & & \\
\hline de 9 à 10,9 & 17 & 4,65 & 37 & 2,25 & 6,96 & 8,5 \\
\hline de 11 à 12,9 & 26 & 4,00 & 28 & 2,37 & 4,45 & 6,7 \\
\hline de 13 à 14,9 & 10 & 5,22 & 25 & 2,52 & 4,12 & 5,7 \\
\hline de 15 à 16,9 & 18 & 5,45 & 27 & 2,93 & 5,04 & 4,7 \\
\hline de 17 à 18,9 & 7 & 5,64 & 26 & 3,89 & 3,85 & 4,2 \\
\hline de 19 à 20,9 & 3 & 4,71 & 17 & 3,28 & 3,03 & 3,6 \\
\hline de 21 à 22,9 & 5 & 4,72 & 24 & 3,09 & 3,76 & 3,6 \\
\hline de 23 à 24,9 & 4 & 4,63 & 9 & 2,80 & 2,98 & 3,0 \\
\hline de 25 à 26,9 & & & 8 & 3,33 & 3,28 & 2,5 \\
\hline de 27 à 28,9 & & & 4 & 2,37 & 3,02 & 2,6 \\
\hline de 29 à 30,9 & & & 1 & 4,01 & 2,28 & 2,5 \\
\hline de 31 à 32,9 & & & 1 & 3,61 & 2,45 & - \\
\hline
\end{tabular}

*Majcen (1979) Érable à sucre dans l'érablière à sucre à bouleau jaune et hêtre à grandes feuilles.

**Fortin (1983) Érable à sucre dans la zone feuillue.

Puisque l'érable à sucre est la plus représentée parmi les quatre essences, il est intéressant de comparer son taux d'accroissement à celui de l'érable à sucre dans l'Outaouais (Majcen 1979) et à celui de l'érable à sucre dans la zone feuillue du Québec (Fortin 1983). Le tableau 5 montre que Lejeune dépasse la zone feuillue du Québec et fait très bonne figure avec l'Outaouais. Par contre, Squatec est nettement inférieur à l'Outaouais et inférieur à la zone feuillue du Québec.

Il convient néanmoins de souligner que le taux de croissance individuel intègre les différences liées à la station et celles liées à la densité des peuplements; alors que le faible taux de Squatec peut être la conséquence d'une faible fertilité stationnelle, la supériorité du taux de Lejeune pourrait être la conséquence des dégagements et des élaircies dans ce peuplement. L'accroissement annuel courant par ha permettra de mieux distinguer la productivité stationnelle de l'effet de la sylviculture.

\section{Comparaison selon la classe de cime.}

II est bien connu que le volume $\mathrm{V}$ et son accroissement annuel courant $\mathrm{dV} / \mathrm{dt}$ suivent respectivement les patrons (a) et (b) de la figure 3. Ces deux patrons induisent nécessairement pour le taux d'accroissement dV/Ndt le patron (c).
Ce rappel étant fait, nous allons tenter de vérifier le patron (c). La figure 4 nous montre qu'il est difficile de trouver une courbe moyenne pour l'ensemble des érables à sucre avec une précision acceptable. II serait fort intéressant pour la sylviculture de pouvoir y distinguer des plages. Chaque plage correspondrait à un groupe d'arbres ayant un potentiel sylvicole particulier. De ce fait, la question qui se pose est la suivante: est-il possible de distinguer les groupes et comment pouvons-nous les distinguer? Pour tenter d'y répondre, nous avons utilisé la notion de classe de cime. Celle-ci se définit comme suit:

Dominant: arbre ayant une cime mieux développée que les voisins. La cime n'a aucune contrainte d'espace dans son développement, elle reçoit la lumière par le dessus et par les côtés.

Codominant: arbre ayant une cime partiellement dévelopée, devant partager son espace vital avec ses voisins dominants et codominants. La cime reçoit la lumière par le haut et un peu par les côtés.

Intermédiaire: arbre ayant une cime étranglée par ses 


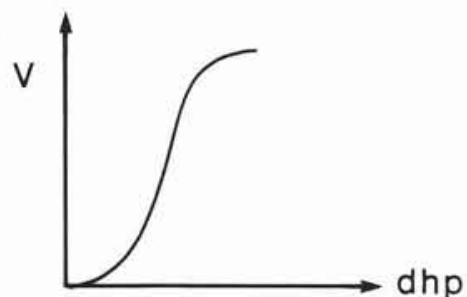

(a)

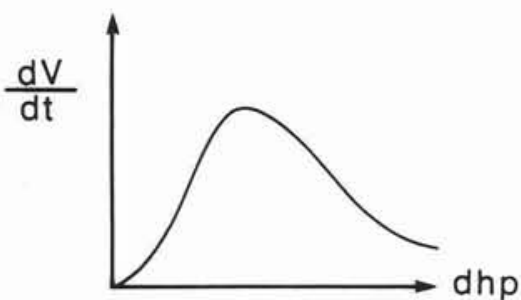

(b)

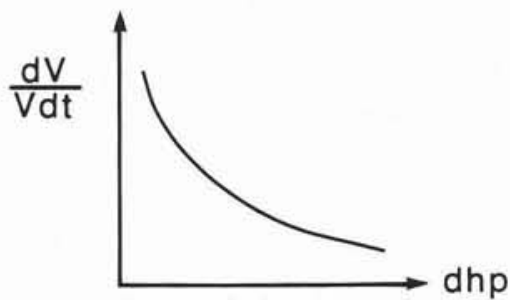

(c)

Figure 3. Patrons du rendement (a), de l'accroissement annuel courant (b) et du taux d'accroissement (c).
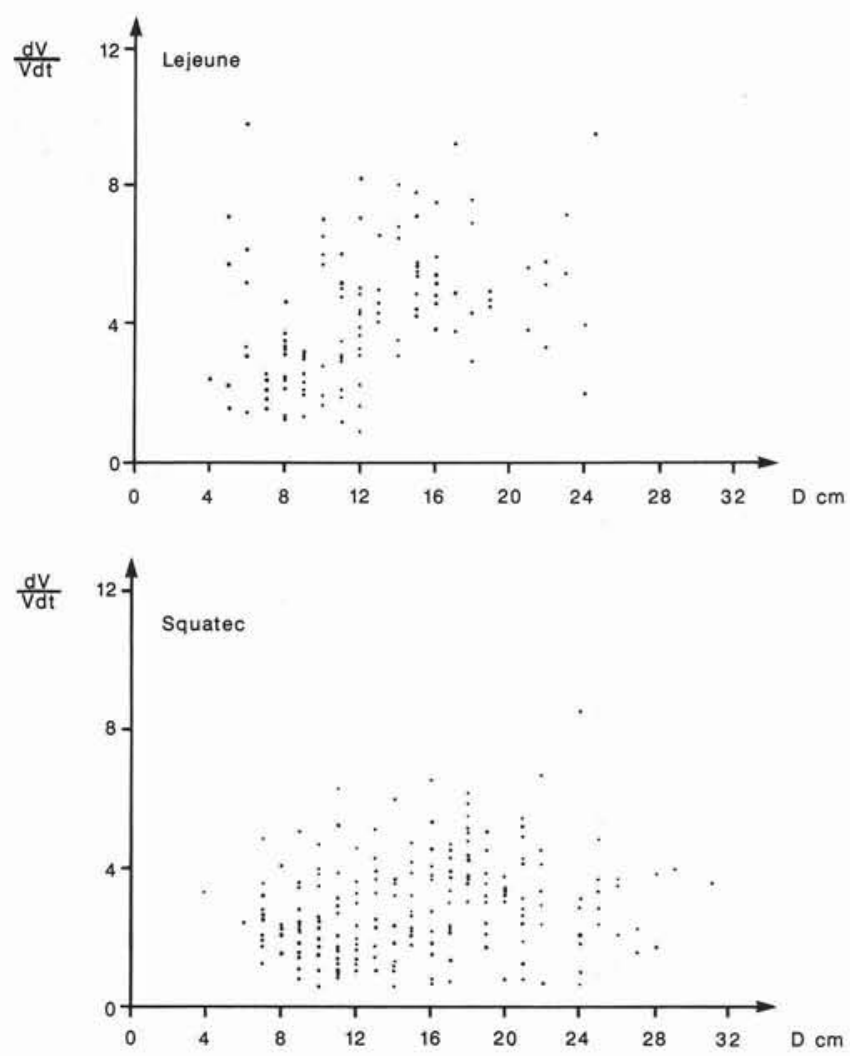

Figure 4. Taux d'accroissement en fonction du dhp, toutes les classes de cime réunies.

voisins, se faufilant dans les espaces inoccupés de ceux-ci. La cime ne reçoit la lumière que par le haut et en petite quantité.

Opprimé: arbre ayant une cime chétive et se développant sous la cime des voisins. Sa cime ne reçoit qu'une lumière indirecte.

À chaque arbre sondé, une classe de cime a été attribuée sur le terrain. Les arbres étant regroupés selon leur classe de cime, les figures 5 et 6 nous montrent que respectivement pour Lejeune et Squatec la tendance de la courbe exponentielle décroissante est relativement plus visible qu'auparavant. Mais les largeurs des plages restent encore larges, comme par exemple le groupe codominant à Squatec qui présente pour le dhp $18 \mathrm{~cm}$, des taux d'accroissement variant de 2 à $7 \%$.

Nous pouvons ici conclure que les classes de cime utilisées représentent certainement un facteur important pour séparer les arbres en groupes homogènes du point du vue de l'accroissement. Mais, puisque les plages demeurent larges, ces classes de cime ne suffisent pas à elles seules à séparer les groupes de façon plus précise. Néanmoins, il faut nous garder à l'esprit que les calculs des accroissements en volume reposent sur seulement deux mesures, le dhp et la longueur d'une carotte et que tout le reste s'obtient par calcul. En reconnaissant que le calcul exact du volume dépend à la fois du dhp, de la hauteur et du coefficient de forme, l'estimation des accroissements en volume, telle qu'effectuée, est certainement entachée d'erreur puisque seule la dimension horizontale est considérée. Cette erreur est d'autant plus grande que l'excentricité des cernes est importante, particulièrement chez les gros diamètres puisqu'une seule carotte a été prise.

\section{Comparaison selon le site:}

II convient de rappeler que la classe de dhp 9 à $19,9 \mathrm{~cm}$ présente le plus grand nombre de tiges dans les deux massifs. De ce fait nous utilisons la moyenne des taux d'accroissement dans cette classe pour caractériser chaque placetteéchantillon. Pour Squatec qui est une érablière à sucre presque pure, le problème du choix des essences ne se pose pas. Mais pour Lejeune, constitué d'une mosaïque d'essences, nous n'avons pas adopté la solution simple consistant à calculer la moyenne des taux d'accroissement de toutes les essences. Nous avons choisi l'essence qui représente plus de $50 \%$ de la surface terrière totale de la placette lors de l'inventaire intégral.

Pour Lejeune (figure 7), nous constatons que:

- les quatres placettes ayant des taux d'accroissement relativement faibles $(3,3,3,4,3,5$ et $3,9 \%)$ forment une concentration se trouvant sur le sommet convexe de la pente (au nord du massif):

- au sud-est (en bas de la figure), il existe une grande zone possédant des taux d'accroissement particulièrement élevés.

Quant à Squatec (figure 8), les taux d'accroissement relativement élevés se présentent, d'une part, pour les placettes-échantillons situées en bas de pente et, d'autre part, pour les placettes-échantillons situées dans les petites cuvettes isolées.

Les prochains résultats de l'analyse des sols, prélevés du pédon creusé dans chaque placette-échantillon, permettront d'étudier les relations entre les propriétés physicochimiques du sol et le taux d'accroissement des arbres. II convient de rappeler que Zarnovican (1985) a commencé à étudier déjà en profondeur ces relations pour les sapinières dans l'unité de gestion des Chics-Chocs.

Notons enfin que nous avons aussi recueilli les données 

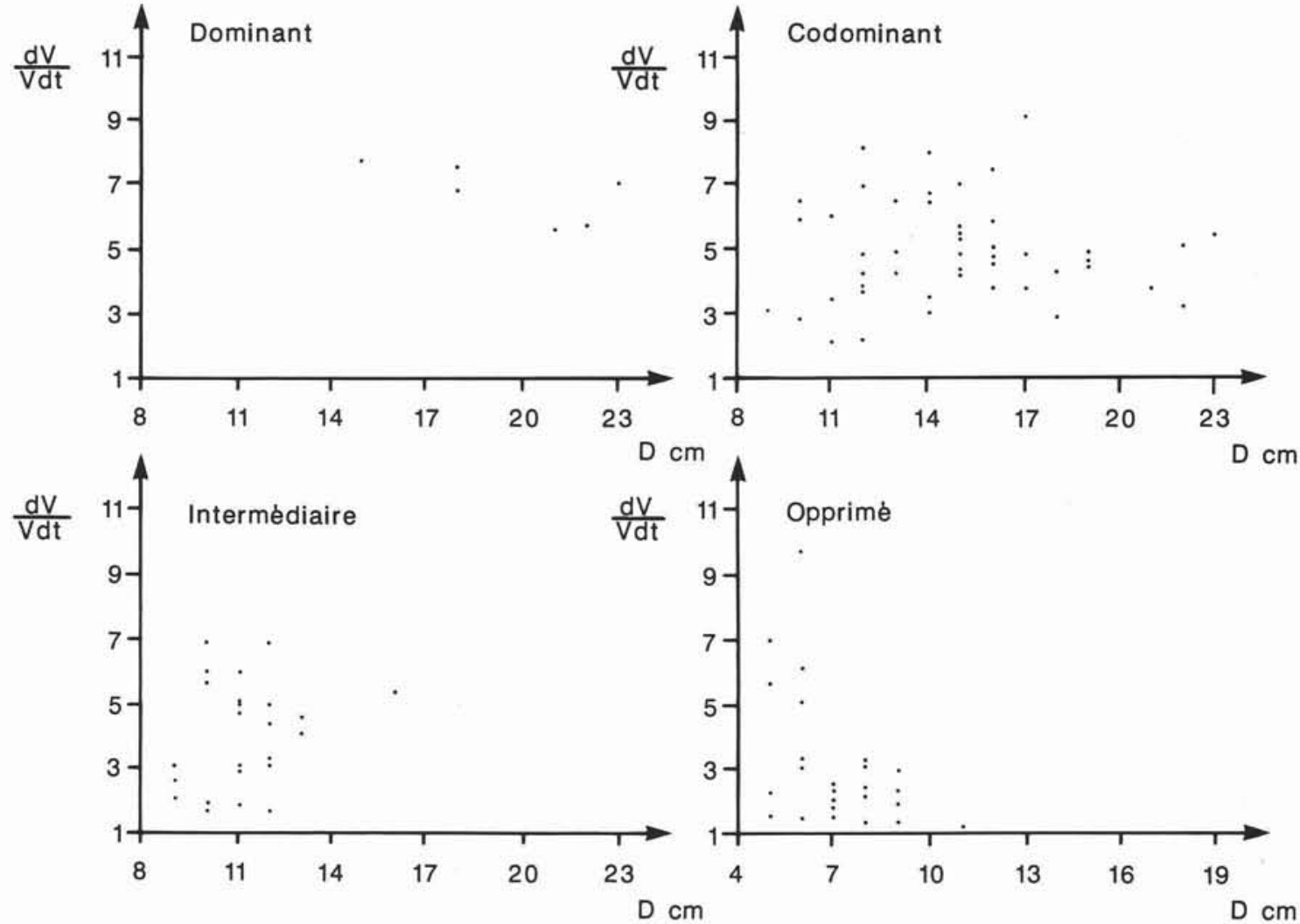

Figure 5. Lejeune: taux d'accroissement en fonction du dhp, les classes de cime séparées.
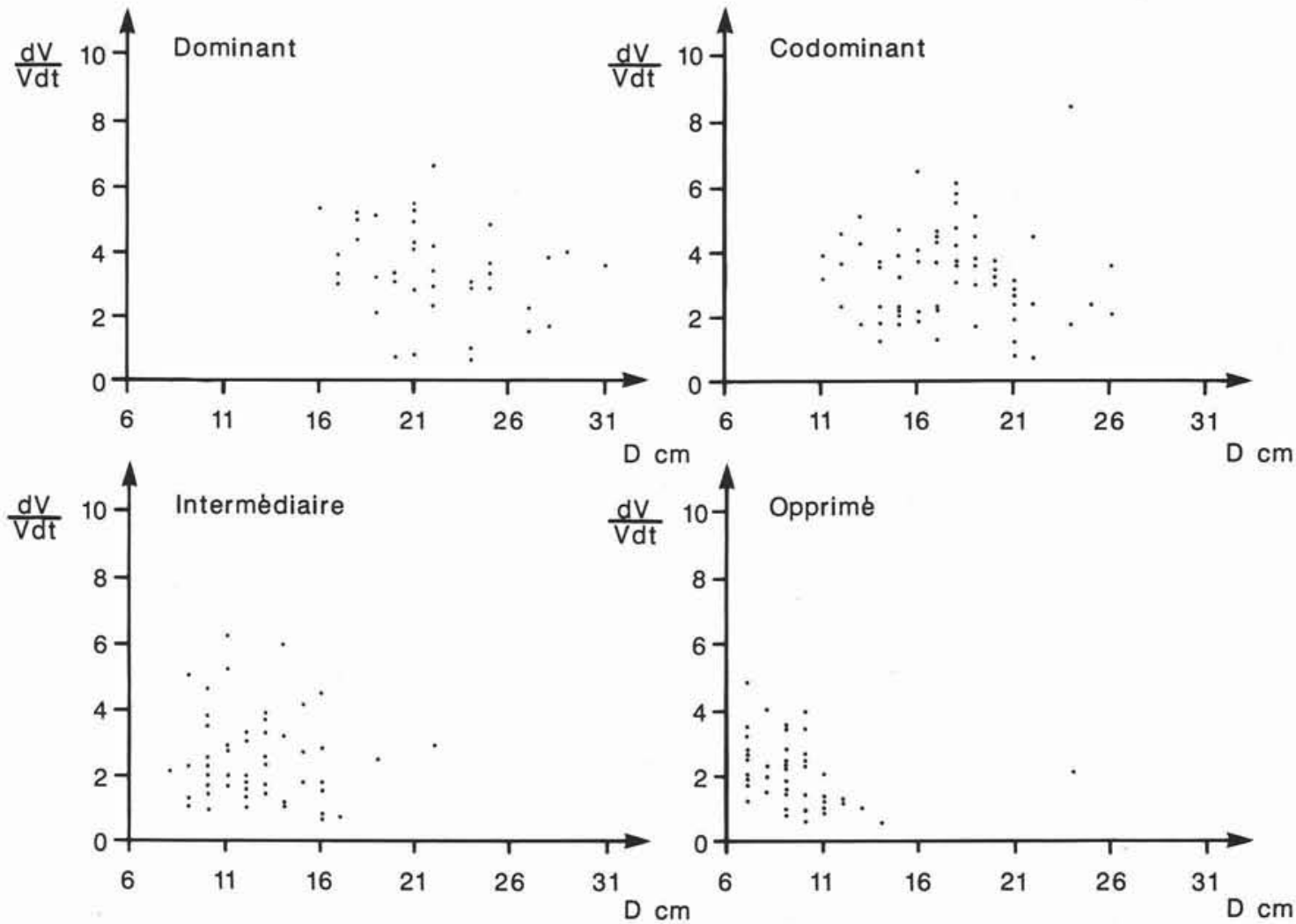

Figure 6. Squatec: taux d'accroissement en fonction du dhp, les classes de cime séparées. 
sur la végétation des strates inférieures au niveau de placettes-échantillons. La méthode d'échantillonnage de cette végétation a été décrite par Zarnovican (1983). Les espèces les plus fréquentes parmi les 49 espèces recencées sont Acer saccharum, Acer pensylvanicum, Acer spicatum, Clintonia borealis, Dryopteris spinulosa, Fagus grandifolia, Maianthemum canadense, Streptopus roseus, Trientalis borealis, Viburnum alnifolium. Parmi ces espèces, Acer spicatum, Clintonia borealis, Maianthemum canadense, Streptopus roseus et Viburnum alnifolium sont plus fréquentes à Lejeune qu'à Squatec. Or ces cinq espèces reflètent un sol relativement frais. Ceci semble représenter le deuxième facteur favorisant l'accroissement à Lejeune par rapport à Squatec, le premier facteur étant la densité des tiges qui est plus faible à Lejeune qu'à Squatec induisant ainsi un accroissement plus élevé à Lejeune qu'à Squatec.

\section{Accroissement annuel courant à l'hectare}

Notons tout d'abord que le calcul de l'accroissement annuel courant à l'hectare se base sur les moyennes des accroissements annuels courants par classes de dhp dont les intervalles sont plus fins que ceux des cinq classes présentées au tableau 6 . Ceci est nécessaire puisque plus l'intervalle de la classe est large, moins la moyenne est fiable (plus la variance est élevée).

L'examen du tableau 6 permet de constater que Lejeune possède un accroissement annuel courant à l'hectare à peu près équivalent à celui de Squatec et ce malgré son nombre de tiges à l'hectare fortement inférieur à celui de Squatec. Notons les accroissements de la classe 9 à $19,9 \mathrm{~cm}$, $3,32 \mathrm{~m}^{3} /$ halan pour Lejeune et $2,50 \mathrm{~m}^{3} /$ halan pour Squatec.

Par rapport à l'Outaouais (Majcen 1979), les accroissements annuels courants à l'hectare (particulièrement ceux de la classe 9 à $19,9 \mathrm{~cm}$ qui représente l'essentiel) sont nettement inférieurs. Ceci est tout à fait prévisible. Mais par rapport aux feuillus tolérants des tables de rendement normal de Plonski (1974) les massifs étudiés font très bonne figure: $4,18 \mathrm{~m}^{3} /$ halan pour Lejeune et $4,50 \mathrm{~m}^{3} /$ ha/an pour Squatec par rapport à $3,4 \mathrm{~m}^{3} /$ halan pour le site 1 âgé de 35 ans.

\section{Qualité des tiges}

Chacun des sept arbres dans chaque placette-échantillon a été examiné. Les arbres morts ou dépérissants sont automatiquement déclassés.

IIs sont 58 à Lejeune et 87 à Squatec, soit respectivement $17,4 \%$ (Lejeune) et $25,3 \%$ (Squatec) du total des arbres examinés.

Le tableau 7 nous montre que les trois classes de qualité représentent chacune le tiers des tiges examinées. En examinant seulement les tiges de qualité bonne (tableau 8 ), leur nombre est de 499/ha à Lejeune et de 358/ha à Squatec. Ces chiffres nous paraissent suffisants pour croire à la rentabilité des coupes d'assainissement qui doivent être réalisées dans les deux massifs.

Tableau 6. Accroissement annuel courant à l'hectare

\begin{tabular}{|c|c|c|c|c|c|c|}
\hline \multirow[b]{2}{*}{$\begin{array}{l}\text { Classes de } \\
\text { dhp en cm }\end{array}$} & \multicolumn{5}{|c|}{ Lejeune } & \multirow{2}{*}{$\begin{array}{l}\text { Squatec } \\
\text { Érable } \\
\text { à sucre }\end{array}$} \\
\hline & $\begin{array}{l}\text { Bouleau } \\
\text { jaune }\end{array}$ & $\begin{array}{l}\text { Érable } \\
\text { rouge }\end{array}$ & $\begin{array}{l}\text { Érable } \\
\text { à sucre }\end{array}$ & Hêtre & $\begin{array}{l}\text { Les quatre } \\
\text { essences }\end{array}$ & \\
\hline \multicolumn{7}{|c|}{ Accroissement annuel courant en $\mathrm{m}^{3} / \mathrm{ha} / \mathrm{an}$} \\
\hline $\begin{array}{l}\text { jusqu'à } 4,9 \\
\text { de } 5 \text { à } 8,9 \\
\text { de } 9 \text { à } 19,9 \\
\text { de } 20 \text { à } 29,9 \\
\text { de } 30 \text { et plus }\end{array}$ & $\begin{array}{c}0,002 \\
0,005 \\
0,782 \\
0,311 \\
0\end{array}$ & $\begin{array}{c}0 \\
0,006 \\
0,593 \\
0,051 \\
0\end{array}$ & $\begin{array}{c}0,041 \\
0,011 \\
1,089 \\
0,196 \\
0\end{array}$ & $\begin{array}{c}0,052 \\
0,020 \\
0,860 \\
0,156 \\
0\end{array}$ & $\begin{array}{c}0,095 \\
0,042 \\
3,324 \\
0,714 \\
0\end{array}$ & $\begin{array}{l}-\overline{011} \\
2,498 \\
1,687 \\
0,305\end{array}$ \\
\hline $\begin{array}{l}\text { Toutes les } \\
\text { classes }\end{array}$ & 1,100 & 0,650 & 1,337 & 1,088 & 4,175 & 4,501 \\
\hline \multicolumn{7}{|c|}{ Nombre de tiges à l'hectare } \\
\hline $\begin{array}{l}\text { jusqu'à } 4,9 \\
\text { de } 5 \text { à } 8,9 \\
\text { de } 9 \text { à } 19,9 \\
\text { de } 20 \text { à } 29,9 \\
\text { de } 30 \text { et plus }\end{array}$ & $\begin{array}{r}10 \\
11 \\
143 \\
19 \\
1\end{array}$ & $\begin{array}{r}13 \\
14 \\
121 \\
5 \\
0\end{array}$ & $\begin{array}{r}273 \\
17 \\
228 \\
14 \\
1\end{array}$ & $\begin{array}{r}77 \\
22 \\
170 \\
18 \\
3\end{array}$ & $\begin{array}{r}373 \\
64 \\
662 \\
56 \\
5\end{array}$ & $\begin{array}{r}738 \\
19 \\
719 \\
147 \\
10\end{array}$ \\
\hline $\begin{array}{l}\text { Toutes les } \\
\text { classes }\end{array}$ & 184 & 153 & 533 & 290 & 1160 & 1633 \\
\hline
\end{tabular}

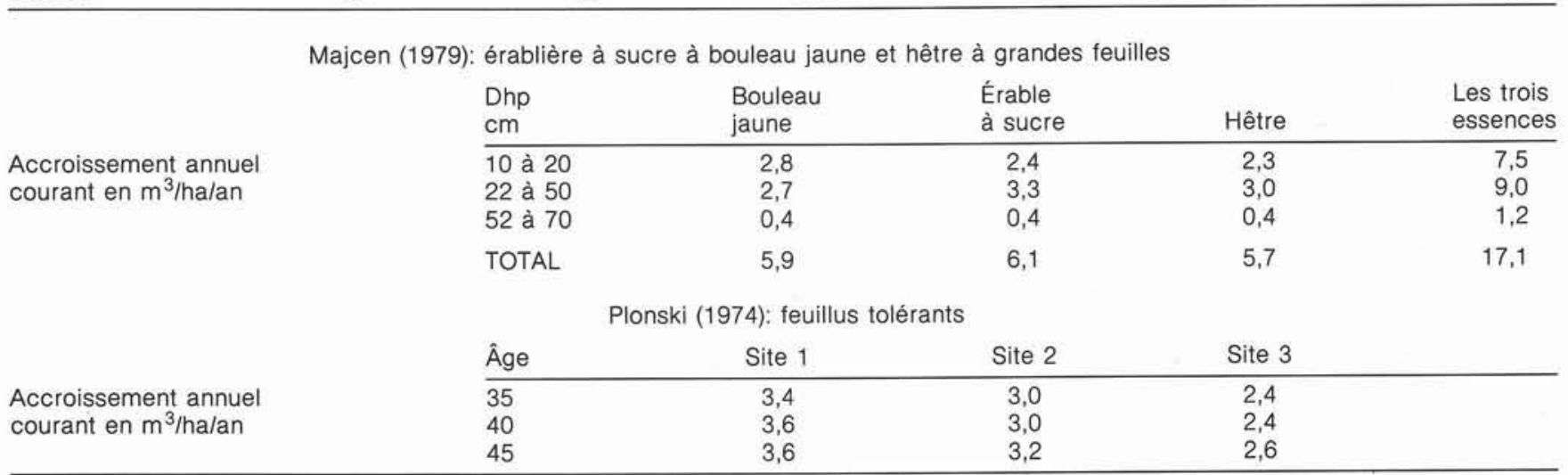




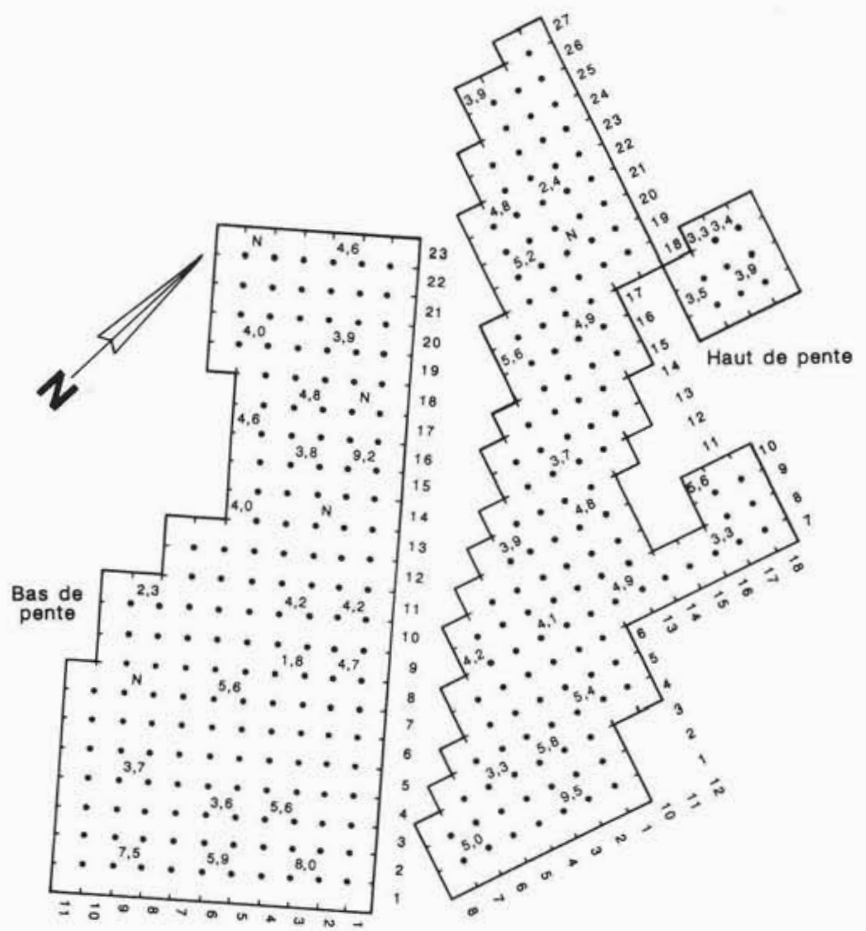

Figure 7. Lejeune: taux d'accroissement au niveau des placettes-échantilions.

\section{Conclusion}

L'accroissement annuel courant en volume des deux massifs au Témiscouata est aussi bon sinon meilleur que ceux des feuillus tolérants dans les tables de rendement normal de Plonski. Le nombre de tiges de qualité bonne paraît suffisant pour la récolte finale. Nous croyons que les deux massifs ont une valeur potentielle pour la production du bois de sciage. De ce fait, nous recommandons que les coupes d'assainissement soient réalisées dans les deux massifs. Ces coupes ont le double but d'assainir les massifs forestiers et de favoriser l'accroissement diamétral des arbres. Ceci est particulièrement urgent à Squatec où l'accroissement en diamètre se trouve ralenti par la trop forte densité des tiges.

Concernant le bouleau jaune, malgré son taux d'accroissement relativement élevé, nous recommandons la prudence pour les scénarios sylvicoles cherchant à le favoriser. En effet, le dépérissement frappe les individus de cette espèce sans égard à leur position sociale.

En rendant relativement plus visible la tendance de courbe exponentielle décroissante du taux d'accroissement en fonction du dhp, la classe de cime est certainement un facteur de différenciation des arbres dans un peuplement en groupes homogènes du point de vue de l'accroissement. Mais, comme les plages des nuages de points demeurent larges, le problème du pouvoir discriminant devrait être approfondi tout en gardant à l'esprit que l'erreur de mesure est une cause importante de cette dispersion des points.

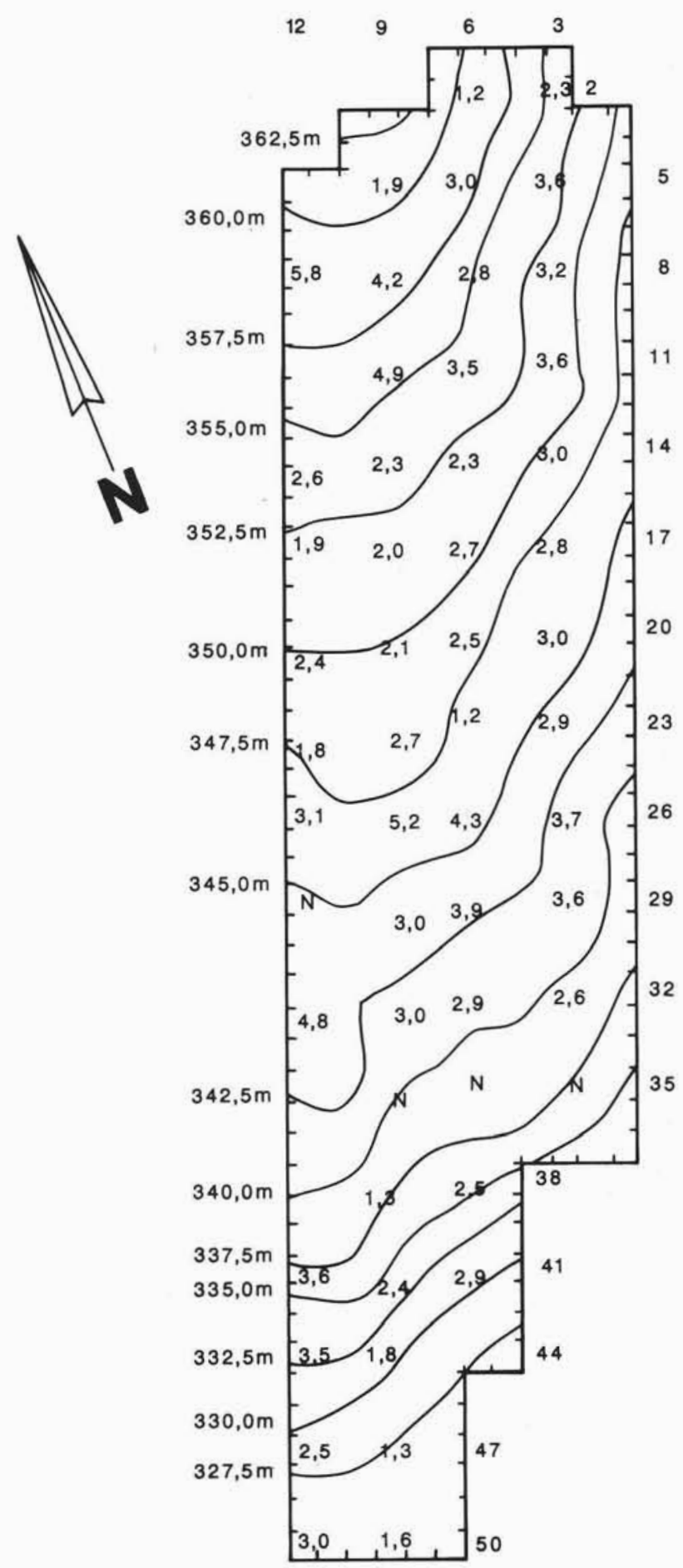

Figure 8. Squatec: taux d'accroissement au niveau des placettes-échantillons.

Le taux d'accroissement est utilisé à Squatec pour permettre une distinction entre placettes plus productives (dans les cuvettes et celles en bas de pentes) et celles moins productives situées sur le sommet. Ce taux met aussi en évidence des différences de comportement spécifique à l'intérieur d'un peuplement homogène. Cependant, le taux d'accroissement individuel n'est pas recommandé pour distinguer les stations productives des stations plus pauvres parce qu'il est fortement influencé par la densité du peuplement. 
Tableau 7. Répartition en pourcentage des arbres selon leur qualité

\begin{tabular}{|c|c|c|c|c|c|c|c|c|}
\hline \multirow[b]{2}{*}{$\begin{array}{l}\text { Classes } \\
\text { dhp en cm }\end{array}$} & \multicolumn{4}{|c|}{$\begin{array}{l}\text { Lejeune: bouleau jaune, érable rouge, } \\
\text { érable à sucre et hêtre }\end{array}$} & \multicolumn{4}{|c|}{ Squatec: érable à sucre } \\
\hline & Médiocre & Moyenne & Bonne & $\begin{array}{l}\text { Les trois } \\
\text { qualités }\end{array}$ & Médiocre & Moyenne & Bonne & $\begin{array}{l}\text { Les trois } \\
\text { qualités }\end{array}$ \\
\hline jusqu'à 4,9 & 14,3 & 85,7 & - & 100 & 18,2 & 81,8 & - & 100 \\
\hline de 5 à 8,9 & 20,8 & 75,5 & 3,8 & 100 & 44,2 & 55,8 & - & 100 \\
\hline de 9 à 19,9 & 33,7 & 22,5 & 43,9 & 100 & 31,2 & 27,3 & 41,6 & 100 \\
\hline de 20 à 29,9 & 45,5 & 18,2 & 36,4 & 100 & 29,2 & 31,3 & 39,6 & 100 \\
\hline de 30 et plus & - & - & - & - & - & 100,0 & - & 100 \\
\hline \multicolumn{9}{|l|}{ Toutes les } \\
\hline classes & 31,2 & 35,5 & 33,3 & 100 & 32,3 & 35,4 & 32,3 & 100 \\
\hline
\end{tabular}

Tableau 8. Nombre de tiges de qualité bonne à l'hectare

\begin{tabular}{lll}
$\begin{array}{l}\text { Nombre de tiges de toute } \\
\text { qualité à l'hectare }\end{array}$ & $\begin{array}{l}\text { Pourcentage des arbres } \\
\text { de qualité bonne }\end{array}$ & $\begin{array}{l}\text { Nombre de tiges de qualité } \\
\text { bonne à l'hectare }\end{array}$ \\
\hline
\end{tabular}

Lejeune: bouleau jaune, érable rouge, érable à sucre et hêtre à grandes feuilles:

jusqu'à 4,9

de 5 à 8,9

de 9 à 19,9

de 20 à 29,0

de 30 et plus

toutes les classes

$\begin{array}{rc}380 & - \\ 68 & 3,8 \\ 672 & 43,9 \\ 59 & 36,4 \\ 6 & -\end{array}$

3.8

43,9

-

295

21

$\frac{1}{499}$

Squatec: érable à sucre:

jusqu'à 4,9

de 5 à 8,9

de 9 à 19,9

de 20 à 29,0

de 30 et plus

toutes les classes

$\begin{array}{cc}- & - \\ -\overline{11} 6 & 300 \\ 39,6 & 58 \\ - & -558\end{array}$

Les relations entre les propriétés physico-chimiques du sol et l'accroissement des arbres devraient être analysées dans le but d'obtenir les résultats qui nous permettront de mieux définir les différentes stations de croissance ainsi que leur frontière dans chaque massif. Toutefois, en reconnaissant la nécessité de connaître en détails le massif forestier pour une sylviculture rationnelle, il ne faudrait pas tomber dans le pointillisme qui s'accorde mal avec une gestion forestière rationnelle.

\section{Références}

Duplat, P. et G. Perrotte. 1981. Inventaire et estimation de l'accroissement des peuplements forestiers. Office National des Forêts, Section Technique (France), $432 \mathrm{p}$.

Fortin, J. 1983. La croissance forestière au Québec. Service de l'inventaire forestier, Ministère de l'Énergie et des Ressources du Québec. 82 p.

Laszlo, M. 1971. Les différentes méthodes d'évaluation de la qualité des tiges. Erdeszeti Kutatasok (Proc. Hung. For. Res. Inst.) 67 (partie 3): 209-222.

Majcen, J. 1979. Relations entre la végétation, les caractères d'habitat et le rendement dans la station forestière d'Argenteuil, Québec. Service de la recherche, Ministère de l'Énergie et des
Ressources du Québec. Rapport interne no 193, 290 p.

Plonski, W.L. 1974. Normal yield tables (metric) for major forest species of Ontario, Ont. Min. Nat. Resour. $40 \mathrm{p}$.

Sonderman, D. 1979. Guide to the measurement of tree characteristics important to the quality classification system for young hardwood trees. USDA For. Serv., Northeast. For. Exp. Stn., Gen. Tech. Rep. NE-54. 11 p.

Ung, C.-H. 1989. Formes des tiges de l'érable à sucre et du hêtre à grandes feuilles dans une jeune futaie selon la position sociale et le temps. Accepté pour publication dans Ann. sci. for.

Zarnovican, R. 1983. Production stationnelle dans les sapinières en Gaspésie, Québec. Ser. can. forêts. Centre rech. for. Laurentides, Sainte-Foy, Québec. Rapp. inf. LAU-X-64.

Zarnovican, R. 1985. Analyse de quelques éléments de la production forestière de l'unité de gestion des Chics-Chocs (partie occidentale). Serv. can. forêts. Centre rech. for. Laurentides, Sainte-Foy, Québec. Rapp. inf. LAU-X-74.

Zarnovican, R. et D. Ouellet. 1987. Peuplier faux-tremble au Témiscouata: allométrie, croissance, production et évolution des prix du bois. Serv. can. forêts. Cent. for. Laurentides, Sainte-Foy, Québec. Rapp. inf. LAU-X-78.

Zarnovican, R. et J. Trencia. 1987. Croissance de l'érable à sucre et du hêtre à grandes feuilles selon la position sociale dans le peuplement. Ann. Sci. For. 44(2): 211-226. 